\title{
Resistência a antifúngicos de Candida tropicalis isoladas no Estado do Ceará
}

\author{
Antifungal resistance of Candida tropicalis isolated \\ in the State of Ceará
}

\author{
Everardo Albuquerque Menezes ${ }^{1}$, Luana Guabiraba Mendes ${ }^{1}$ \\ e Francisco Afrânio Cunha ${ }^{1}$
}

\begin{abstract}
RESUMO
Neste estudo, foi avaliada a resistência a drogas antifúngicas em 51 cepas de Candida tropicalis isoladas de amostras clínicas no Estado do Ceará, Brasil. Resistência antifúngica foi um evento raro no nosso estudo e foi restrita a 3 (5,9\%) das cepas de Candida tropicalis, que exibiram resistência a fluconazol e itraconazol.
\end{abstract}

Palavras-chaves: Candida tropicalis. Agentes antifúngicos. Teste de disco difusão.

\begin{abstract}
In this study, the resistance to antifungal drugs was investigated among 51 strains of Candida tropicalis isolated from clinical samples in the State of Ceará, Brazil. Antifungal resistance was a rare finding in our study and was restricted to three (5.9\%) of the strains of Candida tropicalis. These exhibited resistance to both fluconazole and itraconazole.
\end{abstract}

Key-words: Candida tropicalis. Antifungal agents. Disk diffusion test.

Candida tropicalis é uma agente freqüente de candidemia em hospitais brasileiros, sendo a segunda espécie mais comumente isolada. A infecção por esse agente pode ocorrer em pacientes de todas as idades, mas acomete pacientes adultos e idosos com maior frequiência ${ }^{10}$.

Casuísticas do Brasil confirmam que as três espécies mais prevalentes isoladas de urina em pacientes hospitalizados são: Candida albicans, Candida tropicalis e Candida glabrata. Estes estudos demonstram prevalências de 35,5 a 70\% para Candida albicans; 4,6 a 52,5\% para Candida tropicalis e 7 a 8,8\% para Candida glabrata ${ }^{5112}$.

0 aumento da resistência a antifúngicos alerta para a necessidade do desenvolvimento de estratégias que evitem a sua disseminação entre os fungos, como já ocorreu com as bactérias, que se encontra disseminada e fora de controle ${ }^{1}$. A resistência aos azólicos em cepas de Candida tropicalis é devido ao aumento da expressão do gene $E R G 11$, associada com uma mutação missense nesse gene ${ }^{15}$.

\footnotetext{
1. Departamento de Análises Clínicas e Toxicológicas, Faculdade de Farmácia Odontologia e Enfermagem, Universidade Federal do Ceará, Fortaleza, CE.

Projeto Financiado pelo Conselho Nacional de Desenvolvimento Científico e Tecnológico (CNPq). Processo. 473417/2007.

Endereço para Correspondência: Prof. Everardo A. Menezes. Rua Capitão Francisco Pedro 1210, Rodolfo Teófilo, 60430-370 Fortaleza, CE.

Tel: 5585 3366-8266.

e-mail: menezes@ufc.br

Recebido para publicação em 12/01/2009

Aceito em 14/04/2009
}

Avaliar a resistência a agentes antifúngicos utilizando o teste de microdiluição é trabalhoso e caro. 0 teste de disco difusão com discos de fluconazol utilizando meio Mueller-Hinton ágar com $2 \%$ de glicose e $0,5 \mathrm{mg} / \mathrm{mL}$ de azul de metileno é adequado para procedimentos laboratoriais de triagem da resistência de cepas de Candida. Os resultados devem ser lidos após 24h. Esse método possui boa acurácia e é de baixo custo podendo ser acrescentado na rotina clínica laboratorial ${ }^{6}$.

0 objetivo desse estudo foi avaliar a resistência de Candida tropicalis isoladas no ano de 2008 de amostras clínicas no Ceará pela técnica de disco difusão.

Foram testadas 51 amostras de Candida tropicalis provenientes de pacientes internados no Hospital Geral de Fortaleza. As cepas fazem parte da coleção de Leveduras do Laboratório de Microbiologia da Universidade Federal do Ceará. As amostras estavam congeladas em meio nutriente caldo com $20 \%$ de glicerol e armazenadas a $-20{ }^{\circ} \mathrm{C}$. As cepas foram descongeladas e inoculadas em ágar batata dextrose e incubadas a $35^{\circ} \mathrm{C}$ por $24 / 48 \mathrm{~h}$. Aidentificação das leveduras foi realizada em meio cromógeno Agar Hicrome Candida ${ }^{\circledR}$ (Mumbai-Índia) e confirmada com o teste do microcultivo em ágar arroz com tween $80^{\circ}$.

A sensibilidade das cepas de Candida tropicalis foi avaliada pelo método de disco difusão em ágar em meio Mueller-Hinton adicionado de $2 \%$ glicose e $0,5 \mu \mathrm{g} / \mathrm{mL}$ de azul de metileno de acordo com o protocolo M44-4 do The Clinical and Laboratory Standards Institute (CLSI) ${ }^{2}$. Os discos de antifúngicos utilizados neste estudo foram produzidos em nosso laboratório utilizando drogas padrões: anfotericina B, fluconazol e itraconazol (Sigma 
TABELA 1

Sensibilidade de cepas de Candida tropicalis aos antifúngicos

\begin{tabular}{|c|c|c|c|c|c|c|c|c|c|}
\hline \multirow[b]{2}{*}{ Levedura } & \multirow[b]{2}{*}{ Origem } & \multirow[b]{2}{*}{ Número } & \multicolumn{2}{|c|}{ Anfotericina B (\%) } & \multicolumn{3}{|c|}{ Fluconazol (\%) } & \multicolumn{2}{|c|}{ Itraconazol (\%) } \\
\hline & & & $\mathrm{R}$ & $S$ & $\mathrm{R}$ & SDD & $S$ & $\mathrm{R}$ & $S$ \\
\hline & Urina & 34 & - & 100,0 & 5,9 & - & 94,1 & 5,9 & 94,1 \\
\hline & Sangue & 4 & - & 100,0 & 25,0 & - & 75,0 & 25 & 75,0 \\
\hline Candida & Orofaringe & 5 & - & 100,0 & - & - & 100,0 & - & 100,0 \\
\hline \multirow[t]{4}{*}{ tropicalis } & LBA & 4 & - & 100,0 & - & - & 100,0 & - & 100,0 \\
\hline & $\mathrm{PC}$ & 2 & - & 100,0 & - & - & 100,0 & - & 100,0 \\
\hline & Fezes & 2 & - & 100,0 & - & - & 100,0 & - & 100,0 \\
\hline & Total & 51 & - & 100,0 & 5,9 & - & 94,1 & 5,9 & 94,1 \\
\hline
\end{tabular}

LBA: lavado broncoalveolar, PC: ponta de cateter, R: resistência, SDD: sensível dose dependente, S: sensível.

Chemical Company, St Louis, Mo). o fluconazol e o itraconazol foram diluídos em água e a anfotericina $\mathrm{B}$ foi diluída em dimetilsulfóxido (DMSO). Após a diluição as drogas foram colocadas em discos de papel de filtro de $13 \mathrm{~mm}$ e colocadas na estufa a $35^{\circ} \mathrm{C}$ por $24 \mathrm{~h}$. As concentrações dos antifúngicos nos discos foram: fluconazol $25 \mu \mathrm{g}$, anfotericina B $100 \mu \mathrm{g}$ e itraconazol 30ug. As cepas foram classificadas de acordo com seu perfil de sensibilidade em: sensível (S), sensível dose dependente (SDD) e resistente $(R)^{4}$. Como controles, foram utilizadas as cepas de Candida albicans ATCC 10231, Candida albicans ATCC 14053 e Candida parapsilosis ATCC 22019.

0 perfil de sensibilidade a antifúngicos pode ser observado na Tabela 1. Não foram detectadas neste estudo cepas de Candida tropicalis resistentes à anfotericina B. Foram detectadas $3(5,9 \%)$ cepas de Candida tropicalis resistentes ao fluconazol, duas cepas isoladas de urina e uma isolada de sangue. Foram detectadas 3 (5,9\%) cepas de Candida tropicalis resistentes ao itraconazol, duas cepas isoladas de urina e uma isolada de sangue. As cepas resistentes ao fluconazol também foram resistentes ao itraconazol (Tabela 1).

Algumas cepas de Candida tropicalis são resistentes ao fluconazol e a outros agentes antifúngicos ${ }^{3}$. Em um estudo com 188 cepas de Candida tropicalis isoladas de candidemia apenas uma foi resistente ao fluconazo ${ }^{10}$.

Candida tropicalis foi a causa mais comum de fungemia em estudo realizado com pacientes hematológicos. Doença disseminada foi comum e resultou em elevada mortalidade ${ }^{7}$.

Em um estudo com 100 amostras de leveduras, isoladas de urina, provenientes de Hospital Público Infantil de São Paulo Brasil, no período de 1999-2004, as espécies mais freqüentes foram Candida albicans, seguida de Candida tropicalis, Candida glabrata e Candida parapsilosis ${ }^{14}$.

Um total de 2.949 de Candida spp foram testadas pelo método de disco difusão e pelo método de referência de microdiluição em caldo. Foram obtidos concordância de $92,8 \%$ entre os métodos. 0 teste de disco difusão foi excelente para detectar cepas de Candida spp. resistentes aos antifúngicos ${ }^{13}$.

A resistência a azólicos entre cepas de Candida tropicalis isoladas no estado do Ceará ainda é baixa e o teste de disco de difusão é uma alternativa ao método de referência sendo mais prático e menos oneroso $0^{8}$.

\section{REFERÊNCIAS}

1. Canuto MM, Rodero FG. Antifungal drug resistance to azoles and polyenes. The Lancet Infectious Diseases 2:550-563, 2002

2. Clinical and Laboratory Standards Institute (CLSI). Methods for antifungal disk diffusion susceptibility testing of yeasts; approved standard M44-A. Clinical and Laboratory Standards Institute, Wayne, PA, 1997.

3. Dassanayake RS, Samaranayake YH, Yau JYY, Samaranayake LP. DNA fingerprinting elicited evolutionary trend of oral Candida tropicalis isolates from diverse geographic locals. Indian Journal Medical Microbiology 24:186-194, 2006.

4. Espinel-Ingroff A. Standardized Disk Diffusion Method Yeast. Clinical Microbiology Newsletter 29:97-100, 2007.

5. Kobayashi CC, Fernandes OF, Miranda KC, Sousa ED, Silva MR. Candiduria in hospital patients: a study prospective. Mycopathologia 158: 49-52, 2004.

6. Lee SC, Fung CP., Lee N, See LC, Huang JS, Tsai CJ, Chen KS, Shieh WB. Fluconazole disk diffusion test with methylene blue- and glucose-enriched Mueller-Hinton agar for determining susceptibility of Candida species. Journal Clinical of Microbiology 39:1615-1617, 2001.

7. Leung AYH, Chim CS, Hoy PL, Chengy VCC, Yueny KY, Lie AKW, Au WY, Liang R, Kwong YL. Candida tropicalis fungaemia in adult patients with haematological malignancies: clinical features and risk factors. Journal of Hospital Infection 50: 316-319, 2002.

8. Matar MJ, Ostrosky-Zeichner L, Paetznick VL, Rodriguez JR, Chen E, Rex JH. Correlation between E-test, disk diffusion, and microdilution methods for antifungal susceptibility testing of fluconazole and voriconazole. Antimicrobial Agents and Chemotherapy 47:1647-1651, 2003.

9. Menezes EA, Monteiro MNR, Parente TMA, Cunha FA, Augusto KL, Freire CCF. Frequency and enzymatic activity of Candida albicans isolated from the oral cavity of HIV-positive patients at Fortaleza, Ceará. Jornal Brasileiro de Patologia e Medicina Laboratorial 42: 253-256, 2006.

10. Nucci M, Colombo AL. Candidemia due to Candida tropicalis: clinical, epidemiologic, and microbiologic characteristics of 188 episodes occurring in tertiary care hospitals. Diagnostic Microbiology and Infectious Disease 58: 77-82, 2007.

11. Oliveira RD, Maffei CM, Martinez R. Infecção urinária hospitalar por leveduras do gênero Candida sp. Revista da Associação Médica Brasileira 47: 321-325, 2001.

12. Passos XS, Sales WS, Maciel PJ, Costa CR, Miranda KC, Lemos JA, Batista MA, Silva MRR. Candida colonization in intensive care unit patients' urine. Memórias do Instituto Oswaldo Cruz 100: 925-928, 2005.

13. Pfaller MA, Hazen KC, Messer SA, Boyken L, Tendolkar S, Hollis RJ, Diekema DJ Comparison of results of fluconazole disk diffusion testing for Candida species with results from a central reference laboratory in the ARTEMIS global antifungal surveillance program. Journal Clinical of Microbiology 42:3607-3612, 2004.

14. Silva EH, Ruiz LS, Matsumoto FE, Auler MA, Giudice MC, Moreira D, Szeszs W, Paula CR. Candiduria in a Public Hospital of São Paulo (1999-2004): characteristics of the yeast isolates. Revista do Instituto de Medicina Tropical de São Paulo 49:349-353, 2007.

15. Vandeputte P, Larcher G, Bergés T, Renier G, Chabaise D, Bouchara JP. Mechanisms of azole resistance in clinical isolate of Candida tropicalis. Antimicrobial Agents and Chemotherapy 49:4608-4615, 2005. 